\title{
Gentle Dragging of Reaction Waves
}

\author{
J. Wolff, A. G. Papathanasiou, H. H. Rotermund, and G. Ertl \\ Fritz-Haber-Institut der Max-Planck-Gesellschaft, Faradayweg 4-6, 14195 Berlin, Germany
}

X. Li and I. G. Kevrekidis*

Department of Chemical Engineering, Princeton University, Princeton, New Jersey, 08544

(Received 8 July 2002; published 9 January 2003)

\begin{abstract}
Using a recently realized "addressable catalyst surface" [Science 294, 134 (2001)] we study the interaction of chemical reaction waves with prescribed spatiotemporal fields. In particular, we study how a traveling chemical pulse is "dragged" by a localized, moving temperature heterogeneity as a function of its intensity and speed. The acceleration and eventual "detachment" of the wave from the heterogeneity is also explored through simulation and stability analysis.
\end{abstract}

PACS numbers: 82.40.Bj, 05.45.-a, 82.40.Np, 82.45.Jn

As the elements of spontaneous pattern formation are progressively understood through theory, experimentation, and scientific computation [1], ways to purposefully interact with the coherent structures (pulses and fronts) that constitute the building blocks of spatiotemporal patterns are becoming the focus of extensive research [2,3]. In particular, the stabilization and control of various patterns [3-9] through local, nonlocal, or global feedback, and pattern formation in media with designed heterogeneities [10-12] are the source of novel insights for spatiotemporal dynamics.

To explore such phenomena, we have recently constructed an "addressable catalyst": A focused laser beam, manipulated through computer-controlled mirrors and capable of "writing" spatiotemporal temperature heterogeneity patterns on a metal single crystal catalyst. The loop between this actuation and sensing (both resolved in space and time) through nonintrusive microscopies is then closed through the computer or the experimentalist herself/himself in real time. Our model system is the low-pressure catalytic oxidation of $\mathrm{CO}$ on $\mathrm{Pt}(110)$, a reaction exhibiting well-documented spatiotemporal patterns [13-16], and whose macroscopic modeling has reached an advanced level [17-20].

In this Letter we study the interaction of reactive pulses with a single, spatially coherent but temporally mobile heterogeneity. In particular, we use a temperature heterogeneity, localized in space and steadily moving in time, to "drag" spontaneously isothermally forming reactive pulses and fronts with speeds differing from their natural speed. We examine the shapes acquired by these dragged waves and their limits of stability (that is, the range of dragging speeds for which they can exist). Through computer-aided analysis we examine the nature of the detachment instability, marking the loss of the ability of the heterogeneity to drag a pulse in 1D or 2D. Two possible paths to instability (depending on the linearized spectrum crossing) are detected. We explore the postdetachment transient dynamics and the interactions of a pulse with successive elements of a $1 \mathrm{D}$ periodic, constant speed array of identical heterogeneities. The theme of pulse dragging is currently also being explored in a variety of physically relevant Hamiltonian systems, or weakly perturbed dissipative variations thereof (targeted transfer of pulses in optical media or the motion/displacement of harmonic traps or optical lattices that induces dragging of Bose-Einstein condensates [21,22]). It would be interesting to explore the similarities/differences between these studies and our results.

Experiments were performed in a UHV chamber, equipped with low-energy electron diffraction (LEED), $\mathrm{Ar}^{+}$sputtering, and sample heating (from the back side) via a halogen lamp. The $10 \mathrm{~mm}$ diameter sample was prepared by repeated cycles of $\mathrm{Ar}^{+}$sputtering and $\mathrm{O}_{2}$ treatment at $570 \mathrm{~K}$, and subsequent annealing to $1000 \mathrm{~K}$. Gas supplies for $\mathrm{CO}$ and $\mathrm{O}_{2}$, and pressure gauges, allow for controlled dosing of the reactants. Adsorbate concentration patterns on the surface of the sample are imaged using ellipsomicroscopy for surface imaging (EMSI) [23]. The light of another $\mathrm{Ar}^{+}$laser is focused onto a spot of about $50 \mu \mathrm{m}$ diameter on the surface, causing a local temperature increase of up to $10 \mathrm{~K}$. Using computercontrolled galvanometer mirrors, the focused laser beam can be positioned in $1 \mathrm{~ms}$ with a spatial precision of $5 \mu \mathrm{m}$ anywhere within the viewing field $(1.5 \times$ $1.3 \mathrm{~mm}^{2}$ ). Because of $\operatorname{Pt}(110)$ anisotropy, typical wave speeds depend on the angle the direction of propagation forms with the principal crystal axes: target patterns take elliptical forms.

We use the term "point dragging" for results obtained through constant speed movement of the laser spot focused down to $50 \mu \mathrm{m}$. Since at these conditions and dragging speeds the temperature field response is very fast compared to the time scales of the catalytic reaction dynamics [24], we will assume that the constant speed movement of the focused heat source is effectively modeled by a (approximately Gaussian) moving temperature heterogeneity whose amplitude and width depend on the 
heating power and focus width of the laser beam. This has been qualitatively confirmed through IR camera measurements.

The mechanism of low-pressure $\mathrm{CO}$ oxidation on single crystal surfaces is well established. A standard three-species model [17] describes the adsorption/desorption of $\mathrm{CO}$ and $\mathrm{O}_{2}, \mathrm{CO}$ surface diffusion, the formation of $\mathrm{CO}_{2}$, as well as the surface conversion between a $1 \times 1$ and a missing row $1 \times 2$ structure depending on the $\mathrm{CO}$ coverage,

$$
\begin{aligned}
\frac{\partial u}{\partial t} & =k_{u} s_{u} p_{\mathrm{CO}}\left[1-\left(\frac{u}{u_{s}}\right)^{3}\right]-k_{2} u-k_{3} u v+\nabla \cdot(D \nabla u), \\
\frac{\partial v}{\partial t} & =k_{v} p_{O_{2}}\left[w s_{v 1}+s_{v 2}(1-w)\right]\left(1-\frac{u}{u_{s}}-\frac{v}{v_{s}}\right)^{2}-k_{3} u v, \\
\frac{\partial w}{\partial t} & =k_{5}(f(u)-w),
\end{aligned}
$$

$u$ and $v$ denote the surface coverages of $\mathrm{CO}$ and $\mathrm{O}$, respectively, $w$ is the fraction of the surface area in the $1 \times 1$ phase, $f(u)$ is an experimental fit to the rate of change of the surface structure and takes the following form for a $\mathrm{Pt}(110)$ surface:

$$
f(u)= \begin{cases}0, & u \leq 0.2, \\ -\frac{u^{3}-1.05 u^{2}+0.3 u-0.026}{0.0135}, & 0.2<u<0.5, \\ 1, & u \geq 0.5 .\end{cases}
$$

In the model, the control parameters are the partial pressure of $\mathrm{CO}, \mathrm{O}_{2}$, and the crystal temperature $T$, which enters the rate constants $k_{2}, k_{3}$, and $k_{5}$ through Arrhenius activation: $k_{i}=\left(k^{0}\right)_{i} \exp \left(-E_{i} / R T\right)$. It is through these $k_{i}$ that the moving heterogeneity $T(x, t)$ affects the dynamics. Compared to laser heating, the heat released by the reaction at such low pressures is negligible. Reaction dynamics do not, thus, affect temperature dynamics. In the 1D case (which corresponds to experimentally dragging a front or pulse with a laser line segment) we used heterogeneities with maximum temperature rise ranging from 0.2 to $1 \mathrm{~K}$ and standard deviations of $2 \mu \mathrm{m}$.

Transient simulations in 1D and 2D were performed using the finite element method for the spatial discretization, and the method of lines in time (trapezoidal as well as variable order backward differentiation formula integration using the DASPK [25] software). Steady (traveling) states were computed through Newton-Raphson iteration and pseudo-arclength continuation; a boundary value problem formulation of the 1D steady problem as a periodically forced oscillator in space is also underway [24]. The generalized eigenproblems arising in the study of the stability of these steady states were solved using ARPACK [26]. Since the local temperature rise was small, we ignored the variation (with temperature and concentration) of the phenomenological diffusion coefficient in our model. Taking the detailed dependence of transport on gradients of chemical potentials into account [27] would be necessary for quantitative modeling. Figure 1(a)
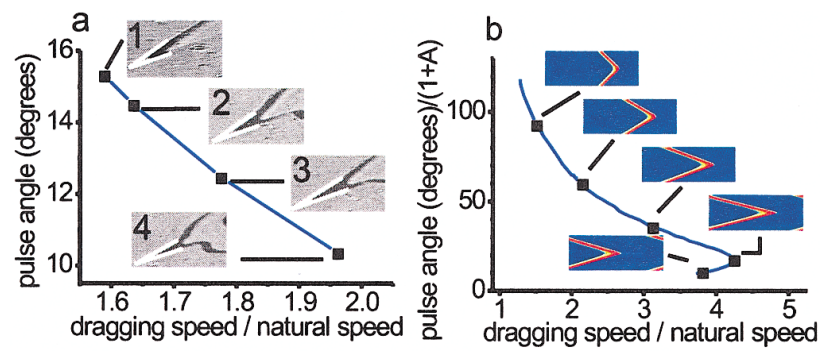

FIG. 1 (color). Experimental (a) and computed (b) dependence of the leading angle of the dragged pulse on the dragging speed (normalized with the natural pulse speed). Experimental conditions: $T=456 \mathrm{~K}, P_{\mathrm{CO}}=3.9 \times 10^{-5} \mathrm{mbar}, P_{\mathrm{O}_{2}}=3.0 \times$ $10^{-4}$ mbar. The dragging direction was about $4^{\circ}$ off the fast diffusion direction. The angle is marked by white lines in (a) in the $0.7 \times 0.4 \mathrm{~mm}^{2}$ insets. Computational parameters: $T=$ $540 \mathrm{~K}, \quad P_{\mathrm{CO}}=4.52 \times 10^{-5} \mathrm{mbar}, \quad P_{\mathrm{O}_{2}}=1.33 \times 10^{-4} \mathrm{mbar}$, and $D_{\mathrm{CO}}=1 \mu \mathrm{m}^{2} / \mathrm{s} ; 180 \times 60 \mu \mathrm{m}^{2}$ domain; boundary conditions: periodic in $x$ and no flux in $y . A$ is used in (b) to visually discriminate the stable $(A=0)$ and the unstable $(A$ proportional to the spot-pulse tip visual distance) branch.

shows experimental results for point dragging of a reacting pulse at four different dragging speeds. At these conditions the natural pulse speed is $30.3 \mu \mathrm{m} / \mathrm{s}$ (respectively, $4.9 \mu \mathrm{m} / \mathrm{s}$ ) along the [1]0] (respectively, [001]) direction. As the dragging speed increases, the angle formed by the pulse becomes increasingly sharper. At some critical dragging speed (here approximately $59 \mu \mathrm{m} / \mathrm{s}$ ) the spot cannot steadily drag the pulse any more; the pulse detaches and gradually returns to its autonomous elliptical shape.

Computer-assisted modeling was used to analyze the dragging phenomenon. Figure 2(a) shows a compendium of one-parameter bifurcation diagrams of dragged onedimensional pulses. The dragging speed is the bifurcation parameter, and color codes the variation with the intensity of the heterogeneity, quantified by the temperature rise at its center. Clearly, there is an interval of "stable dragging," bounded at high speeds by a turning point bifurcation: a stable and an unstable dragged pulse branch collide and disappear. The nature of the bifurcation strongly suggests a single real eigenvalue crossing the imaginary axis through 0 at the critical dragging speed, and the branch turns back, becoming unstable.

Remarkably, two different instability scenarios are found: At low laser spot intensities, an isolated eigenvalue crosses at the turning point [see Fig. 2(b)1,2]. At higher spot intensities an entire "parabola" of eigenvalues, strongly suggestive of continuous spectrum, begins to cross at criticality [see Fig. 2(b)6]; the first eigenvalue (tip) crossing corresponds to the turning point. Subsequent crossings suggest an almost continuous cascade of Hopf bifurcations for the discretized problem.

Continuous spectrum crossing has been associated with the onset of "chemical turbulence" in models of $\mathrm{CO}$ oxidation [28]; this motivates future careful 

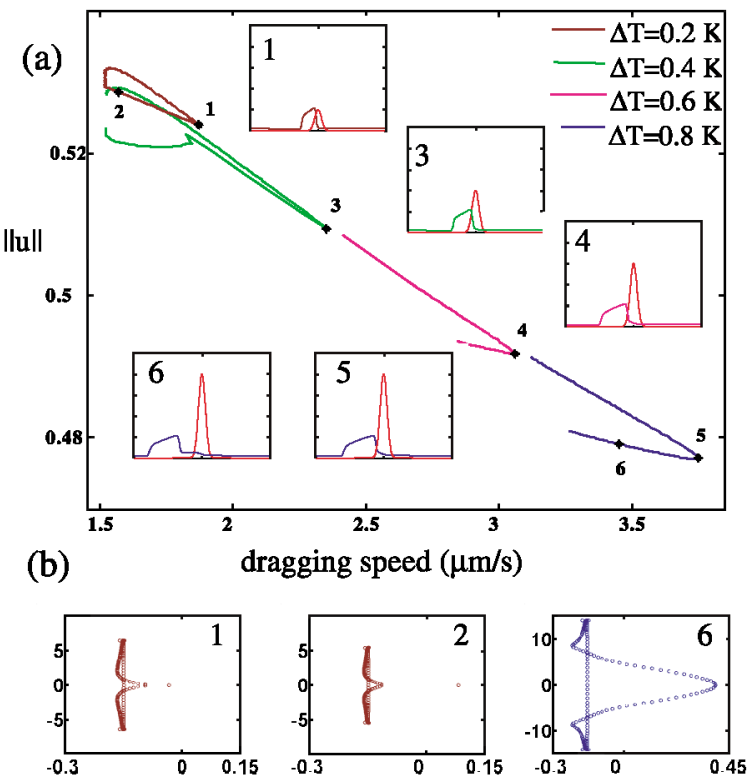

FIG. 2 (color). (a) Computed average CO coverage vs dragging speed for different heterogeneity "strengths" ( $\Delta T$ at the center of the laser spot). Insets show selected oxygen coverage and dragging temperature 1D profiles. Spectra corresponding to points 1,2 (low $\Delta T$ ) and 6 (high $\Delta T$ ) are shown in (b). $T=$ $540 \mathrm{~K}, \quad P_{\mathrm{CO}}=4.52 \times 10^{-5} \mathrm{mbar}, P_{\mathrm{O}_{2}}=1.33 \times 10^{-4} \mathrm{mbar}$, and $D_{\mathrm{CO}}=1 \mu \mathrm{m}^{2} / \mathrm{s} ; 1 \mathrm{D}, 80 \mu \mathrm{m}$ long, periodic domain.

investigation of the "detachment dynamics" of the pulse beyond the turning point, as well as of the dynamics in the neighborhood of the unstable pulses. Notice also on the unstable branch the (visual) distance between the steadily dragged pulse and the (moving) temperature spot. The pulse appears visually connected with the heterogeneity through a region of intermediate oxygen coverage, which decreases in size as the unstable branch approaches the turning point.

At certain conditions of spot intensity and speed in this diagram [Fig. 2(a)], due to the excitability of the underlying medium, the spot becomes capable of generating pulses (target patterns) spontaneously on its own. We should thus differentiate between a "subcritical" spot, incapable of self-initiating pulses, but able to drag a preexisting pulse [as in Fig. 1(a)], and a "supercritical" spot, capable of both initiating and dragging pulses(see Fig. 2A in [2]). A detailed study of the interplay between dragging and the transition from a subcritical to a supercritical spot is currently under investigation.

Two-dimensional simulations are performed in relatively large computational boxes with periodic boundary conditions in the $x$ and no-flux in the $y$ direction. When a pulse detaches from the dragging spot and exits the domain on the left, it enters again on the right, interacting with a subsequent identical laser spot following at a distance given by the periodicity. This "spot array" geometry can give rise to locking and quasiperiodic detachment dynamics. The ordinate in Fig. 1(b) is the apparent angle $\theta$ of the dragged front; for a perfect triangle, asymptotically $\sin (\theta / 2)=u_{1 \mathrm{D}} / u_{\mathrm{drag}}$, the ratio of the natural to the dragging speed. Clearly, the main 1D feature - the turning point and concomitant detachment at high dragging speeds - persists. Here the heterogeneity is supercritical and the detachment involves the crossing of apparent continuous spectrum; a long, thin "tether" connects the heterogeneity with the tip of the pulse. Very narrow in the direction transverse to dragging, this tether is reminiscent of those observed when elastic membranes under tension become subject to strong point forces [29]. Figure 3 samples the richness of "array dragging" pulse detachment dynamics. The pulse detaches from a toofast-moving heterogeneity, is dragged for some time by the following heterogeneity, detaches again, and so on. Depending on the dragging speed these "point-pulse" interactions can be periodic [repeating after a finite number of encounters, Fig. 3(a)] quasiperiodic [never repeat exactly, Fig. 3(b)], and, almost certainly, also chaotic.

Figure 4(a) illustrates experimental supercritical pulse generation-detachment events: the heterogeneity (indicated by an arrow in the first frame) moves too fast to retain the pulses to which it gives birth. Figure 4(b) shows a computed detachment sequence from a supercritical moving heterogeneity. The generated pulse detaches, and after some time another pulse is created. With periodic array dragging, a bewildering variety of dynamics arises from the interaction of newly generated and "previously detached" pulses. Figure 4(c) (experimental) and Fig. 4(d) (computational) show space-time $(x-t)$ plots transversely to the dragging direction, taken at a constant distance from (and comoving with) the laser spot. The detachment (just beyond critical dragging) appears symmetric in the (isotropic) simulation of Fig. 4(d), but

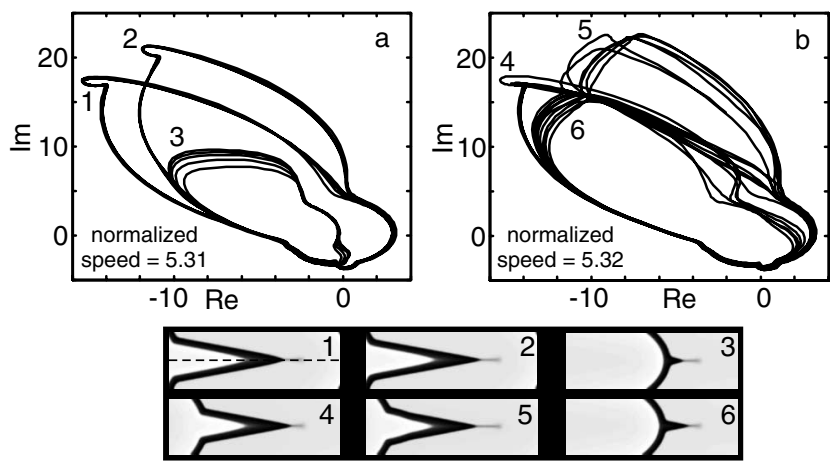

FIG. 3. Computed types of pulse detachment [phase portraits, (a) and (b), and snapshots, (1)-(6)] at different dragging speeds. In (a) and (b) we plot the real versus the imaginary part of the first fast Fourier transform component of the computed $\mathrm{CO}$ coverage along the line marked in (1). Representative $u(x, y)$ snapshots at the onset of the detachment are marked in (a) and (b) and shown in (1)-(6). The remaining conditions are as in Fig. 1(b). 


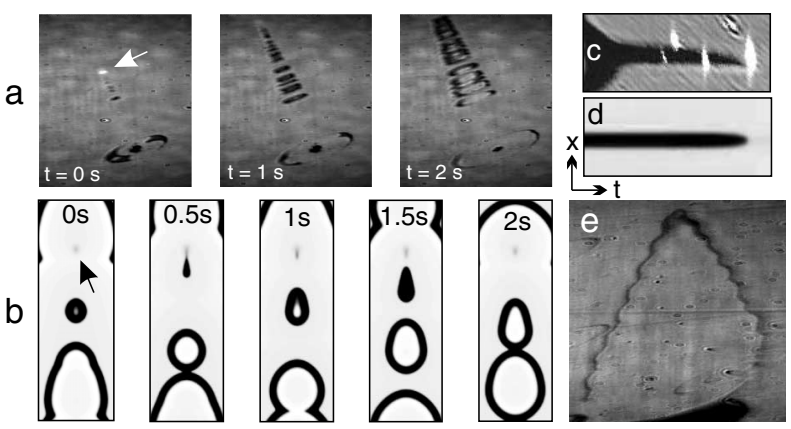

FIG. 4. (a) Experimental (size: $1 \times 1.2 \mathrm{~mm}^{2}, T=493 \mathrm{~K}$, $P_{\mathrm{CO}}=7.0 \times 10^{-5} \mathrm{mbar}, P_{\mathrm{O}_{2}}=3.0 \times 10^{-4} \mathrm{mbar}$ ), and (b) computational snapshots, comoving with the laser spot, of pulse initiation/detachment, (c) $x$ - $t$ plot of transient pulse detachment beyond point 4 in Fig. 1(a), and (d) a computational analogue. (e) "Snake" dragging (size: $1 \times 1 \mathrm{~mm}^{2}, \quad T=$ $\left.501 \mathrm{~K}, P_{\mathrm{CO}}=7.3 \times 10^{-5} \mathrm{mbar}, P_{\mathrm{O}_{2}}=3.0 \times 10^{-4} \mathrm{mbar}\right)$.

a deviation from this symmetry, due to experimental anisotropy, is clear in Fig. 4(c). The tip of the pulse deviates towards the direction of faster front movement after detaching from the laser spot. A laser spot is arguably the simplest 2D "dragging function." Figure 4(e) shows a different such dragging function: the laser spot movement has two components, a constant speed vertical translation, superimposed with a horizontal oscillation. The dragged pulse assumes a "snakelike" form with ripples along its sides.

We presented experimental and computational results pertaining to the interaction of coherent reactiondiffusion patterns (waves, pulses) with moving, spatially localized heterogeneities. In our simulations, these heterogeneities were localized temperature "spots" or "lines," which were experimentally realized through a computercontrolled, mobile, focused laser spot. The observations include the progressive sharpening of the dragged front and the existence of a critical dragging speed beyond which the front detaches from the spot. This is a turning point bifurcation, and two underlying mechanisms were identified through computation, involving either discrete or continuous spectrum crossing. Analogies of this phenomenology to other fields (optical trap Bose-Einsteincondensation manipulation, point force induced membrane tether formation) were noted. Current research focuses on tether formation, on the interaction of pulses and fronts with slow-moving heterogeneities, and the effects of more spatially complex dragging functions, both in the subcritical regime - when they are not capable of spontaneous pattern initiation-and in the supercritical one.
This work was partially supported by NSF, AFOSR, Princeton PPST (X. Li), a Humboldt Forschungspreis (I. G. K.) and the EEC Marie Curie Program (A. G. P.). We thank P.G. Kevrekidis and B. Malomed for helpful discussions.

*Corresponding author.

Email address: yannis@ @rinceton.edu

[1] M. C. Cross and P. C. Hohenberg, Rev. Mod. Phys. 65, 851 (1993).

[2] J. Wolff et al., Science 294, 134 (2001).

[3] S. Kadar, J. C. Wang, and K. Showalter, Nature (London) 391, 770 (1998).

[4] V. Petrov et al., Nature (London) 361, 240 (1993).

[5] O. Steinbock, V. Zykov, and S.C. Muller, Nature (London) 366, 322 (1993).

[6] V. S. Zykov, A. S. Mikhailov, and S. C. Muller, Phys. Rev. Lett. 78, 3398 (1997).

[7] M. Kim et al., Science 292, 1357 (2001).

[8] V. S. Zykov et al., Phys. Rev. E 65, 026206 (2002).

[9] T. Sakurai et al., Science 296, 2009 (2002).

[10] M. D. Graham et al., Science 264, 80 (1994).

[11] M. Pollmann et al., Phys. Rev. Lett. 86, 6038 (2001).

[12] O. Steinbock, P. Kettunen, and K. Showalter, Science 269, 1857 (1995).

[13] K. C. Rose et al., Surf. Rev. Lett. 5, 1233 (1998).

[14] H. H. Rotermund, Surf. Sci. Rep. 29, 267 (1997).

[15] R. Imbihl, Physica (Amsterdam) 188A, 34 (1992).

[16] H. H. Rotermund et al., Nature (London) 343, 355 (1990).

[17] K. Krischer, M. Eiswirth, and G. Ertl, J. Chem. Phys. 96, 9161 (1992).

[18] M. Bär, Ph.D. thesis, Freie Universitaet, Berlin, 1993.

[19] A. v. Oertzen et al., J. Phys. Chem. B 104, 3155 (2000).

[20] X. Li et al., Chaos 12, 190 (2002).

[21] A. Smerzi et al., Phys. Rev. Lett. 89, 170402 (2002).

[22] B. P. Anderson and M. A. Kasevich, Science 282, 1686 (1998).

[23] H. H. Rotermund et al., Science 270, 608 (1995).

[24] X. Li, M. Sheintuch, and I. G. Kevrekidis (to be published).

[25] P. N. Brown, A. C. Hindmarsh, and L. R. Petzold, SIAM J. Sci. Comput. 15, 1467 (1994).

[26] R. B. Lehoucq, D. C. Sorensen, and C. Yang, ARPACK Users's Guide (SIAM, Philadelphia, 1998).

[27] V. P. Zhdanov and B. Kasemo, J. Stat. Phys. 90, 79 (1998); V. Pereyra, G. Zgrablich, and V. P. Zhdanov, Langmuir 6, 691 (1990).

[28] M. G. Zimmermann et al., Physica (Amsterdam) 110D, 92 (1997).

[29] D. K. Fygenson, J. F. Marko, and A. Libchaber, Phys. Rev. Lett. 79, 4497 (1997); T. R. Powers, G. Huber, and R. E. Goldstein, Phys. Rev. E 65, 041901 (2002). 